\title{
Different mechanisms of mitotic instability in cancer cell lines
}

\author{
ALEXANDRA KLEIN ${ }^{1}$, KLAUS D. ZANG ${ }^{1}$, WOLF-INGO STEUDEL ${ }^{2}$ and STEFFI URBSCHAT ${ }^{2}$ \\ ${ }^{1}$ Institute of Human Genetics, Saarland University, Building 60; ${ }^{2}$ Department of Neurosurgery, \\ Saarland University, Building 90.5, D-66421 Homburg/Saar, Germany
}

Received April 24, 2006; Accepted June 28, 2006

\begin{abstract}
Chromosomes of human malignant tumours display not only structural recombinations but also a wide variety of mostly non-random numerical aberrations. However, only little is known about the mechanisms leading to recurrent aneuploidies. We therefore investigated whether the malsegregation of specific chromosomes is due to a defect of the mitotic spindle apparatus. We analyzed mitoses of cell lines of six gliomas and of one breast carcinoma by combined immunohistochemistry and fluorescence in situ hybridization for nondisjunction of chromosomes 7, 8, 10, 12, 17, and 18 and observed three different phenomena. i) Five of six glioma cell lines showed a bipolar spindle but displayed a chromosome-specific malsegregation of all chromosomes studied with high but significantly different frequencies. Chromosomes 7 and 8 showed non-disjunction in about 75 and $50 \%$, respectively. Although chromosomes 10, 12, 17, and 18 displayed equal separation during mitosis in $72,86,73$, and $78 \%$, respectively, a relevant percentage of an average of $24 \%$ of dividing cells showed even malsegregation of these chromosomes. ii) Only one of the glioma cell lines displayed multipolar spindles in one-third of the investigated cells resulting in non-specific aneuploidy. iii) The breast cancer cell line MCF7 displayed a bipolar spindle, but high frequencies of non-disjunction of all six investigated chromosomes but without preferential loss or gain of specific chromosomes indicating a different mechanism of chromosome malsegregation. In a small percentage of mitoses the chromatids of both homologous chromosomes were not separated mimicking the mechanism in the first meiotic division. This mechanism of double non-disjunction, not detectable by conventional cytogenetic analysis, procreates cell clones with genomic separation for particular chromosomes resulting in homozygosity for mutations which had been present heterozygously in the initial tumour cells.
\end{abstract}

Correspondence to: Dr Steffi Urbschat, Neurooncology, Building 60, Saarland University, D-66421 Homburg/Saar, Germany E-mail: hgsmur@uniklinik-saarland.de

Key words: mitotic instability, spindle apparatus, chromosomal malsegregation, aneuploidy, non-disjunction, glioma, MCF7

\section{Introduction}

Chromosomal instability resulting in aneuploidy is a typical biological feature of malignancies and has been considered to be a major driving force in multistep carcinogenesis (1). In fact, cancer is characterized by random or non-random numerical and structural aberrations of chromosomes. One of the most striking manifestations of chromosomal instability in cancer cells is the variation observed between the chromosomal changes of different cells even within the same tumour, i.e., clonal evolutions due to intratumoral genetic micro-heterogeneity $(2,3)$. The biological consequence can be alterations in copy number of one or more genes, changes in gene expression, or changes in gene structure leading to structurally altered proteins. Aneuploidy and chromosomal instability (CIN) are often linked to the progressive development of high-grade, invasive tumours, and a high degree of aneuploidy is often correlated with poor prognosis (4).

Abnormalities in the chromosomal segregational apparatus are very likely to play a role in chromosomal instability. These include centrosomal defects, defects in kinetochore microtubule attachment, and disturbed movement of the chromosomes to the spindle poles. While it is likely that the consequences of all of these mechanisms contribute to carcinogenesis, the extent to which each is involved in a given tumour is still largely uncharacterized (5).

It is well known that glioblastoma multiforme is characterized by intratumoral heterogeneity as to both histomorphology and genetic changes. This tumour entity displays, beyond several structural chromosome alterations, a wide variety of numerical aberrations. The most common are monosomy 10 and trisomy 7 . In a recent study we screened tumour tissue sections of human glioblastomas and observed a high percentage of aneuploid cells micro-heterogeneously distributed in the tumours (6). Loeper and collegues assessed with FISH the intratumoral distribution pattern of numerical aberrations of the chromosomes 7, 8, 10, 12, 17, and 18 . Interestingly, for chromosomes 10 and 17 only monosomic and disomic cells were detected, for chromosome 7 only disomic and trisomic (polysomic) cells occurred, whereas chromosomes 8, 12, and 18 displayed monosomic, disomic as well as trisomic cells (6). Both, the high frequency of the different types of aneuploidy and also the quantitative differences of aneuploidy for the chromosomes studied, were unexpected.

With respect to these previous observations, the aim of our present study was to differentiate between the mechanisms 
Table I. Karyotypes of the investigated cell lines. ${ }^{\text {a }}$

\begin{tabular}{ll}
\hline Tumour & Karyotype \\
\hline T3868 & $86,<4 \mathrm{n}>, \mathrm{XX}, \mathrm{hsr}(\mathrm{X})(\mathrm{p} 22) \mathrm{x} 2,-1,-2,-5,+7,+7,+7,-10,-10,-13,-15,-16,+20,+20,+\operatorname{mar}[4]$ \\
TX3868 & $72-73<4 \mathrm{n}>, \mathrm{X}, \mathrm{hsr}(\mathrm{X})(\mathrm{p} 22) \times 2,-2,-3,-4,-6,-6,-8,-9,-10,-10,-11,-12,-13,-14,-15,-15,-17,-18,-18,-20,-21,+1-2 \mathrm{mar}[\mathrm{cp} 6]$ \\
T4795 & $70-83, \mathrm{XY},<3 \mathrm{n}>,-10,-11,+1-7 \mathrm{mar}, \mathrm{dmin}$ \\
T4797 & $47, \mathrm{XX},+7[\mathrm{cp} 4]$ \\
T5135 & $70-86,<4 \mathrm{n}>, \mathrm{cx}, \mathrm{XXYY,dup}(1)(\mathrm{q} 25-44) \times 2,-3,-4,-5,+7,+7,+\operatorname{der}(7),-8,-8,-10,-10,-11,-12,-13,-13,-14,-14, \operatorname{der}(14),-16,-17,-18,-19,-19,-20,-20$, \\
& $+10-15 \mathrm{mar}, \mathrm{dmin}[\mathrm{cp} 15]$ \\
T5913 & $50-53, \mathrm{XX}, \mathrm{cx},+5,+5,+7,+7,+10,+10,+\operatorname{dmin}$ \\
MCF7 & $60-70,-\mathrm{X},-\mathrm{X}, \operatorname{der}(\mathrm{X}) \mathrm{t}(\mathrm{X} ; 3)(\mathrm{p} 22 ; \mathrm{p} 21), \operatorname{del}(1)(\mathrm{q} 32), \operatorname{der}(1) \mathrm{t}(1 ; 22)(\mathrm{q} 12 ; \mathrm{q} 11),+\operatorname{der}(1) \mathrm{t}(1 ; 22)(? ; ?),+\operatorname{der}(2) \mathrm{t}(2 ; 12)(\mathrm{q} 33 ; \mathrm{q} 21),-3, ? \mathrm{del}(4)(\mathrm{p} 11), \mathrm{t}(5 ; 7)$ \\
& $(\mathrm{p} 10 ; \mathrm{q} 10),-6, \operatorname{ins}(7 ; 20)(\mathrm{p} 14 ; ?), \mathrm{t}(8 ; 22)(\mathrm{q} 12 ; \mathrm{q} 11),+\mathrm{t}(8 ; 19 ; 12)(? ; ? ; ?),+\operatorname{der}(8) \times 3,-9,-9,-11,-12,+\operatorname{der}(13)(?) \times 2,+\operatorname{der}(14)(?),-15,-15, \operatorname{del}(16)(\mathrm{q} 22)$, \\
& $+17,-18, \operatorname{der}(20) \mathrm{t}(2 ; 20)(\mathrm{q} 31 ; \mathrm{p} 21),-21, \operatorname{der}(22)(?), \operatorname{der}(22) \mathrm{t}(6 ; 22)(? ; \mathrm{q} 11),+\operatorname{der}(22) \mathrm{t}(16 ; 22)(\mathrm{q} 13 ; \mathrm{q} 13),+2 \mathrm{mar}[\mathrm{cp} 6]$
\end{tabular}

${ }^{a}$ All cell lines, except T4797, are aneuploid and show a complex karyotype. G-banding was performed according to standard procedures (22) and karyotypes were described in accordance with ISCN (23).

underlying these phenomena. Therefore, we visualized the mitotic spindle apparatus immunohistochemically with a monoclonal anti $\alpha$-tubulin antibody. Simultaneously we performed fluorescence in situ hybridization with centromere-specific probes. Because of methodical limitations the analysis was confined to chromosomes 7, 8, 10, 12, 17, and 18 according to the described previous studies. We scrutinized 6 primary glioma cell lines because of the high rate of aneuploidy described in this tumour entity, and additionally the permanent breast cancer cell line MCF7, which is known to display a complex karyotype with numerous structural but also numerical chromosomal aberrations.

\section{Materials and methods}

Cell lines. The primary glioma cell lines T3868, TX3868, T4795, T4797, T5135 and T5913 were established in our laboratory from primary tumours by standard techniques (7). The commercial breast cancer cell line MCF7 was obtained from the American Type Culture Collection (ATCC HTB-22, ATCC, Rockville, MD). Cells were cultured in DMEM with L-glutamin (BioWhittaker, Belgium), 1\% MEM (Gibco Life Technologies, UK) and $10 \%$ fetal calf serum at $37^{\circ} \mathrm{C}$ in a humidified atmosphere containing $5 \% \mathrm{CO}_{2}$. For combined immunohistochemistry and fluorescence in situ hybridization cells were grown on glass slides and fixed with methanol for 10 min at $-20^{\circ} \mathrm{C}$.

Combined immunohistochemical analysis and fluorescence in situ hybridization. To visualize the mitotic spindle, the slides were subjected to indirect immunofluorescence as follows: After fixation in ice-cold methanol for $10 \mathrm{~min}$ and blocking unspecific binding sites with PBS containing 5\% normal goat serum and $5 \%$ bovine serum albumin at room temperature for $30 \mathrm{~min}$, slides were incubated with a monoclonal anti $\alpha$-tubulin antibody (Sigma, Deisenhofen, Germany) at $37^{\circ} \mathrm{C}$ for $40 \mathrm{~min}$. Slides were washed in a PBS/0.02\% Tween-20 solution. Detection of the primary antibody was performed by incubating the slides with a $\mathrm{Cy} 3$ conjugated goat anti-mouse IgG (Dianova, Hamburg, Germany) for another $40 \mathrm{~min}$. Afterwards, cells were washed in a PBS $/ 0.02 \%$ Tween-20 solution, dehydrated in ethanol, and air-dried.

Chromosome numbers were determined by FISH analysis in dividing cells showing mitotic spindle poles visualized by immunofluorescence. FISH analysis was performed using centromeric probes specific for chromosomes 7, 8, 10, 12, 17, 18 as follows: After immunohistochemical staining, one biotin labelled DNA probe specific for the subcentromeric heterochromatin regions of the investigated chromosomes was added to each slide. Cells fixed on slides were denatured together with the DNA probes at $80^{\circ} \mathrm{C}$ for $12 \mathrm{~min}$, and incubated overnight at $37^{\circ} \mathrm{C}$ in a humidified chamber. After hybridization, each slide was washed sequentially for $10 \mathrm{~min}$ with $2 \mathrm{X} \mathrm{SSC}$ at $43^{\circ} \mathrm{C}, 15$ min with $57 \%$ formamide at $43^{\circ} \mathrm{C}$, and 5 min with $4 \mathrm{X} \mathrm{SSC}$ at room temperature. To block unspecific binding sites, slides were incubated with 4X SSC containing $5 \%$ bovine serum albumin for $30 \mathrm{~min}$ at room temperature. Probe detection was performed by incubating the slides with a fluorescein streptavidin antibody (Vector Laboratories, Burlingame, CA) for $30 \mathrm{~min}$ at $37^{\circ} \mathrm{C}$. To amplify the detected signals, slides were incubated with biotinylated anti-streptavidin (Vector Laboratories) and again with the fluorescein streptavidin antibody for another $30 \mathrm{~min}$ at $37^{\circ} \mathrm{C}$. Afterwards, and between the detection steps, cells were washed in $4 \mathrm{X} \mathrm{SSC} / 0.1 \%$ Triton. Then slides were dehydrated in ethanol, air-dried and counterstained with DAPI/Antifade (Vector Laboratories).

Evaluation. Image acquisition was performed by use of a fluorescence microscope Olympus AX70 (Hamburg, Germany) 
Table II. Different pattern of chromosomal distribution in cancer cell lines.

\begin{tabular}{|c|c|c|c|c|c|c|c|c|c|c|c|c|c|c|c|c|}
\hline & \multicolumn{12}{|c|}{ Tumour and chromosomal distribution } & \multirow{2}{*}{\multicolumn{2}{|c|}{ T5913 }} & \multirow{2}{*}{\multicolumn{2}{|c|}{ MCF7 }} \\
\hline & \multicolumn{2}{|c|}{ T3868 } & \multicolumn{2}{|c|}{ TX3868 } & \multicolumn{2}{|c|}{ T4795 } & \multicolumn{2}{|c|}{ T4797 } & \multicolumn{2}{|c|}{ T5135 } & \multicolumn{2}{|r|}{$\Sigma$} & & & & \\
\hline & Equal & Unequal & Equal & Unequal & Equal & Unequal & Equal & Unequal & Equal & Unequal & Equal & Unequal & Equal & Unequal & Equal & Unequal \\
\hline \multicolumn{17}{|c|}{ Bipolar spindel apparatus } \\
\hline \multicolumn{17}{|c|}{$\begin{array}{l}\text { Chromo- } \\
\text { some no. }\end{array}$} \\
\hline 7 & $1 / 6$ & $5 / 6$ & $3 / 10$ & $7 / 10$ & $3 / 11$ & $8 / 11$ & $2 / 6$ & $4 / 6$ & $3 / 15$ & $12 / 15$ & $12 / 48$ & $36 / 48(75 \%)$ & $10 / 15$ & $5 / 15$ & $9 / 16$ & $7 / 16$ \\
\hline 8 & $6 / 9$ & $3 / 9$ & $4 / 8$ & $4 / 8$ & 6/11 & $5 / 11$ & $6 / 10$ & $4 / 10$ & 6/11 & 5/11 & $27 / 48$ & $21 / 48(44 \%)$ & $3 / 4$ & $1 / 4$ & $10 / 15$ & $5 / 15$ \\
\hline 10 & $4 / 6$ & $2 / 6$ & $7 / 10$ & $3 / 10$ & $11 / 14$ & $3 / 14$ & $3 / 4$ & $1 / 4$ & $13 / 19$ & $6 / 19$ & $38 / 53$ & $15 / 53(28 \%)$ & $7 / 9$ & $2 / 9$ & $4 / 6$ & $2 / 6$ \\
\hline 12 & $9 / 9$ & - & $9 / 10$ & $1 / 10$ & $7 / 9$ & $2 / 9$ & $8 / 10$ & $2 / 10$ & $9 / 11$ & $2 / 11$ & $42 / 49$ & $7 / 49(14 \%)$ & $5 / 5$ & - & $10 / 16$ & $6 / 16$ \\
\hline 17 & $5 / 8$ & $3 / 8$ & $7 / 10$ & $3 / 10$ & $7 / 10$ & $3 / 10$ & $7 / 8$ & $1 / 8$ & 7/9 & $2 / 9$ & $33 / 45$ & $12 / 45(27 \%)$ & 9/11 & $2 / 11$ & $11 / 16$ & $5 / 16$ \\
\hline 18 & $7 / 9$ & $2 / 9$ & $8 / 9$ & $1 / 9$ & $8 / 11$ & $3 / 11$ & $4 / 4$ & - & $5 / 8$ & $3 / 8$ & $31 / 40$ & $9 / 40(22 \%)$ & $6 / 6$ & - & $11 / 15$ & $4 / 15$ \\
\hline \multicolumn{17}{|c|}{ Multi-polar spindel apparatus } \\
\hline \multicolumn{17}{|c|}{$\begin{array}{l}\text { Chromo- } \\
\text { some no. }\end{array}$} \\
\hline 7 & - & - & - & - & - & - & - & - & - & - & - & - & - & $6 / 6$ & - & - \\
\hline 8 & - & - & - & - & - & - & - & - & - & - & - & - & - & $2 / 2$ & - & - \\
\hline 10 & - & - & - & - & - & - & - & - & - & - & - & - & - & $3 / 3$ & - & - \\
\hline 12 & - & - & - & - & - & - & - & - & - & - & - & - & - & $4 / 4$ & - & - \\
\hline 17 & - & - & - & - & - & - & - & - & - & - & - & - & - & $1 / 1$ & - & - \\
\hline 18 & - & - & - & - & - & - & - & - & - & - & - & - & - & $4 / 4$ & - & - \\
\hline
\end{tabular}

Values of more than $30 \%$ are shown in bold to elucidate the differences between the different cell lines and chromosomes.

using a software by Metasystems GmbH (Altlussheim, Germany). We analyzed an average of 10 mitoses for each chromosome and each tumour. The position of chromosomes was analyzed better directly under the microscope than on digital images. Therefore, we present additionally a scheme for each displayed anaphase or telophase cell, in which the localization of the spindle poles and the chromosomal distribution is mapped. We evaluated only mitotic anaphase or telophase cells if all spindle poles were clearly visible in one focus plane, so that equal and unequal chromosomal distribution could clearly be distinguished. Thereby, very small and particularly laterally reversed signals were interpreted as chromatids, while large signals were considered to be whole chromosomes consisting of the heterochromatin blocks of both chromatids.

\section{Results}

Mitotic chromosomal segregation in glioma cell lines. We investigated six glioma cell lines for mitotic segregation of chromosomes 7, 8, 10, 12, 17, and 18. All cell lines, except T4797 with only trisomy 7 , showed a complex karyotype (Table I). Five cell lines (T3868, TX3868, T4795, T4797, T5135) showed a bipolar spindle apparatus, and one cell line (T5913) displayed supernumerary spindle poles in about one-third of the analyzed cells. Spindle poles and microtubules of all analyzed cell lines appeared to be intact as far as determinable by immunohistochemical analysis.
Mitotic malsegregation of chromosomes 7 and 8 in gliomas displaying a bipolar spindle apparatus. The glioma cell lines displaying a bipolar spindle apparatus showed comparable frequencies of chromosomal malsegregation during mitosis (Table II). Therefore, cells of these five cases were combined and analyzed together.

Surprisingly, we found unequal distribution of chromosomes 7 in very high frequencies of about $75 \%$ of the investigated cells (36/48). This novel observation indicates that the chromatids were not separated correctly and pulled to the opposite spindle poles, but showed asymmetrical distribution. Fig. 1a illustrates this unequal separation for a mitosis of the glioma cell line T5135. The mitotic cell shows four signals for chromosome 7 near one spindle pole but only one near the opposite pole. From the karyotype this glioma cell line is known to have six copies of chromosome 7 in nearly all cells (Table I). From the number and size of the displayed signals, it is very likely that they represent whole chromosomes with two chromatids. However, two chromatids can only be distinguished at the very left of the chromosomes in Fig. 1a, the other chromatids are not in the same focus plane. Altogether in $60 \%$ (24/40) of the investigated mitoses of these five cell lines we detected a non-disjunction of the chromatids of chromosome 7 .

Chromosome 8 was separated equally in only $27 / 48$, i.e. $56 \%$ of the investigated cells. Fig. 1b displays a mitotic cell of the glioma cell line T5135 with an asymmetrical distribution of chromosomes 8 . This cell displays three chromosomes 8 , 


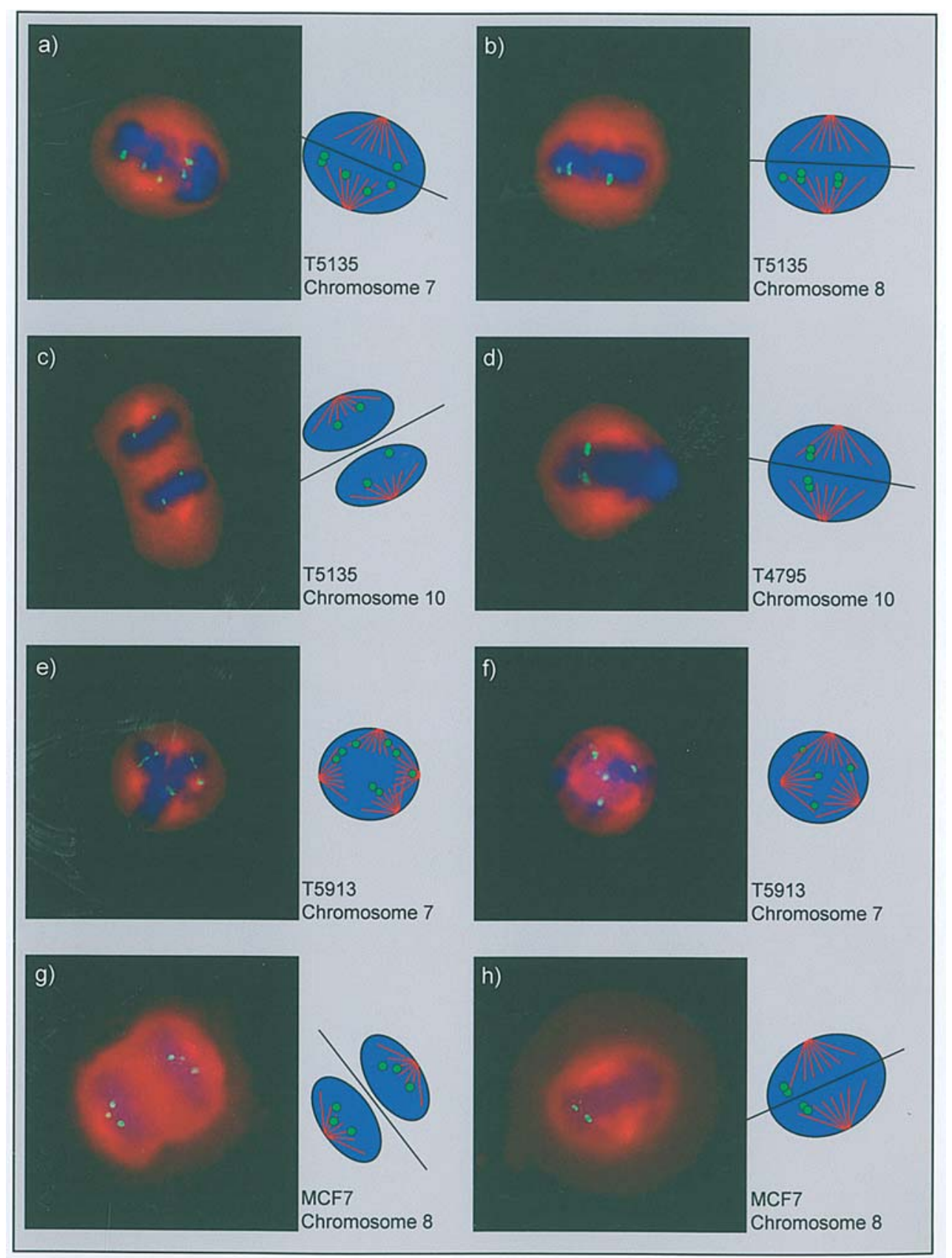

Figure 1. Mitotic chromosomal segregation in tumour cell lines. (a-d) The glioma cell lines T3868, TX3868, T4795, T4797, and T5135 showed a similar pattern of chromosomal malsegregation while the bipolar spindle apparatus appeared to be intact. (a) Unequal distribution of chromosomes 7 each consisting of two chromatids in T5135. (b) Asymmetrical separation of chromosomes 8 each consisting of two chromatids visible as double signals for two of three chromosomes. (c and d) Symmetrical distribution of chromosomes 10 in T5135 and T4795. The telophase cell displays the correct separation of two chromatids to each daughter cell. The anaphase cell of T4795 shows the distribution of whole chromosomes each consisting of two chromatids. (e and f) Distribution of chromosomes 7 in cells of T5913 with tetrapolar respectively tripolar spindle apparatuses. All spindle poles seem to be intact, because they all develop microtubules which allow chromosomes to migrate to the respective spindle pole. Because of the multipolar spindle apparatus, correct chromosomal distribution during mitosis is hampered. (e) The separation of distinct chromatids is shown; while (f) very likely displays whole chromosomes consisting of two chromatids that are distributed as can be seen from chromosome copy numbers shown in Table I. ( $\mathrm{g}$ and $\mathrm{h}$ ) diplay symmetrical mitotic segregation of chromosomes 8 in the MCF7 cell line. The telophase cell in (g) shows three chromatids distributed to each daughter cell. (h) A symmetrical separation of whole chromosomes 8. Non-disjunction of sister chromatids is clearly shown by double signals in FISH-analysis. Magnification x600.

two of them showing a split signal, which are all pulled to one spindle pole. A missing disjunction of the sister chromatids of one or more chromosomes 8 was observed on the basis of visible double signals and the cytogenetically detected chromosome number (Table I) in 17/43 (40\%) mitoses.

For chromosomes 10, 12, 17, and 18 of the five glioma cell lines with a bipolar spindle we found malsegregation in a lower but also high percentage of investigated mitoses. Asymmetrical distribution was observed in $28 \%(15 / 53)$ of cells for chromosome 10, in 14\% (7/49) for chromosome 12 , in $27 \%(12 / 45)$ for chromosome 17 , and in $22 \%(9 / 40)$ of cells for chromosome 18 (Table II). Fig. 1c and d show equal distribution of chromosomes 10 in mitoses of the cell lines T5135 (c) and T4795 (d). In the telophase cell in Fig. 1c two 
Table III. Percentage of mitoses of five glioblastoma cell lines with a bipolar spindle showing asymmetrical distribution of chromosomes $7,8,10,12,17$, and 18 according to Fig. 2b and $\mathrm{d}$.

\begin{tabular}{ll}
\hline Chromosome 7 & $75 \%(36 / 38)$ \\
Chromosome 8 & $44 \%(21 / 48)$ \\
Chromosome 10 & $28 \%(15 / 53)$ \\
Chromosome 12 & $14 \% \quad(7 / 49)$ \\
Chromosome 17 & $27 \%(12 / 45)$ \\
Chromosome 18 & $22 \% \quad(9 / 40)$ \\
\hline
\end{tabular}

Table IV. Percentage of mitoses displaying non-disjunction of sister chromatids of one or more homologous chromosomes $7,8,10,12,17$, and 18 according to Fig. $2 \mathrm{c}$ and $\mathrm{d}$ in five glioblastoma cell lines with a bipolar mitotic spindle. ${ }^{a}$

\begin{tabular}{ll}
\hline Chromosome 7 & $60 \%(24 / 40)$ \\
Chromosome 8 & $40 \%(17 / 43)$ \\
Chromosome 10 & $43 \%(13 / 30)$ \\
Chromosome 12 & $47 \%(23 / 49)$ \\
Chromosome 17 & $60 \%(21 / 35)$ \\
Chromosome 18 & $54 \%(19 / 35)$ \\
\hline
\end{tabular}

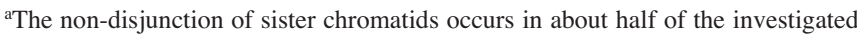
mitoses displaying no significant differences. The high frequency of non-disjunction increases the probability of missegregation of both homologous chromosomes of one chromosome pair mimicking the mechanism of a first meiotic division.

separated chromatids are pulled to each spindle pole. Fig. 1d displays a mitotic cell with a symmetrical distribution also of the chromosomes 10. Remarkably however, in this cell two chromosomes with two chromatids are separated indicating a double non-disjunction resulting in two daughter cells each with isodisomy for one of the two homologous chromosomes 10 . This phenomenon of non-disjunction of chromatids was detected in 13/30 cells for chromosome 10 , in 23/49 cells for chromosome 12, in 21/35 cells for chromosome 17 and in $19 / 35$ cells for chromosome 18 . The results of the five glioblastoma cell lines with a bipolar spindle are summarized in Tables III and IV.

Mitotic malsegregation in cells displaying supernumerary spindle poles. In one of the glioma cell lines (T5913) we observed supernumerary spindle poles in about one-third of the analyzed mitoses (Table II and Fig. 1e and f). The tri- and multipolar spindles appeared to be functional, as all spindle poles formed microtubules that allowed chromosomes to migrate to a spindle pole, but prevented equal distribution of chromatids. In two-third of the mitoses of this tumour we observed bipolar spindles, however also with unequal distribution of the investigated chromosomes in $20 \%(10 / 50)$ of the mitoses (Table II). Fig. 1e and f show examples of the distribution of chromosomes 7 in multipolar mitoses of the
Table V. Percentage of mitoses displaying asymmetrical distribution of chromosomes 7, 8, 10, 12, 17, and 18 according to Fig. $2 b$ and $d$ in the MCF7 cell line.

\begin{tabular}{ll}
\hline Chromosome 7 & $44 \%(7 / 16)$ \\
Chromosome 8 & $33 \%(5 / 15)$ \\
Chromosome 10 & $33 \%(2 / 6)$ \\
Chromosome 12 & $38 \%(6 / 16)$ \\
Chromosome 17 & $31 \%(5 / 16)$ \\
Chromosome 18 & $27 \%(4 / 15)$ \\
\hline
\end{tabular}

glioma cell line T5913 displaying four respectively three spindle poles. The cytogenetic analysis of T5913 revealed four chromosomes 7 in most of the cells (Table I). Therefore, the signals in Fig. 1e very likely represent chromatids of chromosome 7 being pulled to the spindle poles. The four signals for chromosome 7 in Fig. If very likely represent whole chromosomes with two chromatids. Comparing the signals for chromosomes 7 in Fig. 1e and f, signals in the tripolar cell (Fig. 1f) appeared larger than signals in Fig. 1e), supporting the assumption that the signals represent single chromatids 7 in (Fig. 1f). Non-disjunction of chromatids was found in 22/44 mitoses with bipolar spindles and in 9/18 mitoses with multipolar spindles with all investigated chromosomes being affected.

Mitotic chromosomal segregation in the breast cancer cell line MCF7. We detected that all six analyzed chromosomes were also separated unequally in a high percentage of mitoses (Tables II and V). Asymmetrical separation was found in $7 / 16$ cells for chromosome 7 , in $5 / 15$ cells for chromosome 8 , in $2 / 6$ cells for chromosome 10 , in $6 / 16$ cells for chromosome 12 , in $5 / 16$ cells for chromosome 17 , and in $4 / 15$ cells for chromosome 18. All mitoses showed normal bipolar spindles. Fig. 1g and $\mathrm{h}$ show symmetrical distribution of chromosomes 8 to each spindle pole. The telophase cell in Fig. 1g elucidates the correct separation of the chromatids to the daughter cells during mitosis. The six signals likely represent chromatids, because the MCF7 cell line contains three chromosomes 8 . Fig. 1h shows also symmetrical distribution of chromosomes 8 . However, this mitosis reveals a double nondisjunction of both homologous chromosomes with two chromatids visible as small doubled signals. Altogether, we detected non-disjunction of the chromatids for all investigated chromosomes $(7,8,10,12,17$, and 18$)$ in $35 / 64$ of the investigated mitoses.

Table II summarizes the patterns of chromosomal malsegregation in all investigated cell lines. Values of more than $30 \%$ are shown in bold type. Taken together we found three different kinds of chromosomal malsegregation representing different mechanisms leading to chromosomal instability.

First, five of six analyzed glioma cell lines showed a bipolar spindle apparatus and a similar pattern of mitotic chromosomal malsegregation. They displayed unequal distribution of chromosomes 7 in the majority of investigated cells and of 


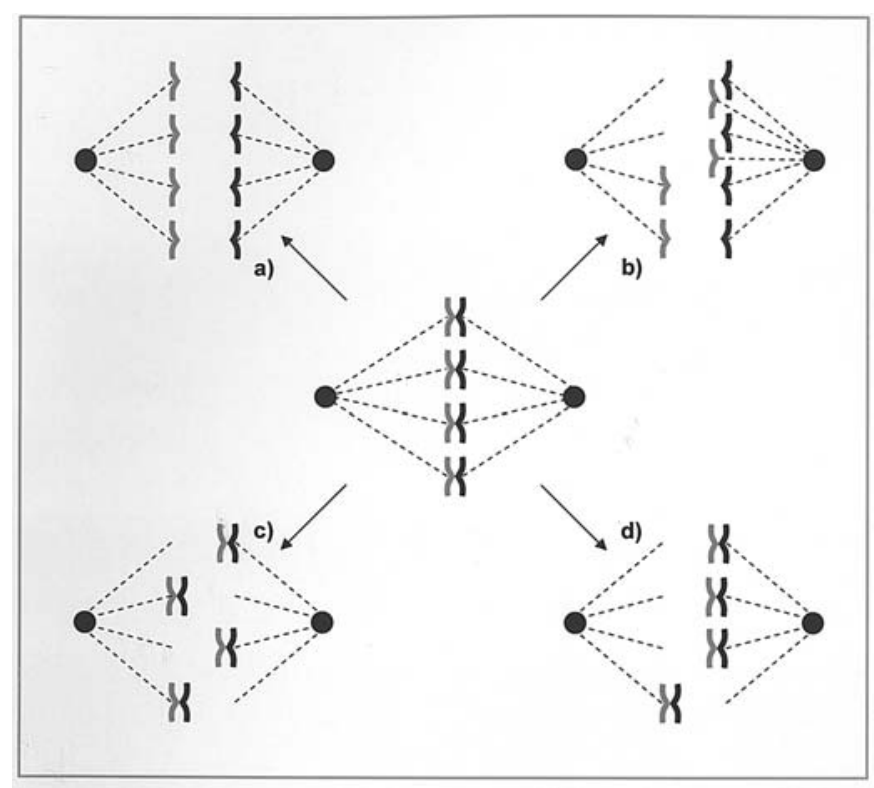

Figure 2. Schematic presentation of the different kinds of mitotic segregation observed in cells showing a bipolar spindle apparatus. (a) Correct separation of chromatids resulting in a symmetrical distribution. (b) Asymmetrical distribution of separated chromatids. (c and d) Non-disjunction of sister chromatids resulting in an apparently symmetrical (c) or in an asymmetrical distribution (d)

chromosomes 8 in about half of the mitoses. Chromosomes $10,12,17$, and 18 displayed symmetrical separation in the majority of the analyzed cells.

Second, one glioma cell line showed only in about two-third of the cells a bipolar spindle apparatus with symmetrical distribution of all analyzed chromosomes on an average of $80 \%$ of the mitoses. Nearly one-third of the analyzed cells displayed supernumerary spindle poles preventing a correct chromosomal distribution.

Third, the breast cancer cell line MCF7 showed a bipolar spindle apparatus and an equal as well as an unequal distribution during mitosis for all investigated chromosomes.

Altogether we detected in all investigated tumour cell lines and for all analyzed chromosomes a non-disjunction of the sister chromatids in a high percentage of cells, so that whole chromosomes were distributed to the daughter cells during mitosis. This phenomenon was found in cells both with symmetrical as well as asymmetrical chromosomal separation.

\section{Discussion}

As early as in 1914, Theodor Boveri described disturbed mitosis and quantitative chromosomal changes in cancers, and forwarded the theory that malignancies of mammalian tissues result from an abnormal chromosomal constitution (8). He suggested inappropriate centrosome duplication and multipolar mitosis to be related to the chromosome abnormalities seen in cancer, in modern terms, chromosomal instability. Genetic instability appears at two distinct levels. In a subset of tumours, the instability is observed at the nucleotide level, i.e. as base substitutions, deletions, or insertions of a few nucleotides (NIN, nucleotide-excision repair associated instability; or MIN, microsatellite instability) resulting in changes of function of single genes. In other cancers, the instability is observed at the chromosome level, resulting in losses and gains of whole chromosomes or parts of them (CIN, chromosomal instability) $(1,9)$. Both effects of the CIN- and of the MIN-phenotype in tumour cells increase the gene dosage of growth-promoting genes, such as oncogenes, or decrease the gene dosage of negative regulators, such as tumour suppressor genes. Cahill et al, reported that the presence of CIN in colon cancer cell lines is associated with dysfunction of the mitotic spindle checkpoint (10). Potential causes of CIN are defects of the mitotic machinery needed for a correct segregation of chromosomes. Examples are failure of spindle attachment to the kinetochore, abnormal centrosome formation, or failure of cytokinesis (11). Abnormal centrosomes are commonly seen in a wide range of cancers including lung, breast, prostate, brain, and colon cancers (12-15). Centrosome amplification drives chromosomal instability in breast tumour development (16) and colorectal cancer cell lines (17). Shono et al, described a strong positive correlation between cells with multiple centrosomes and cells with multipolar mitotic spindles, suggesting a functional involvement of aberrant centrosomes in spindle disorganisation and chromosome missegregation (18). Furthermore, merotelic kinetochore orientation (i.e., the kinetochore is connected to microtubule bundles coming from both spindle poles) is a major mechanism of aneuploidy that is not under control of the mitotic spindle checkpoint $(19,20)$.

Although there are many studies focusing on centrosomal defects resulting in CIN, only little is known about the role of the mitotic spindle apparatus itself in chromosomal missegregation. Therefore, our aim was to analyze tumour cell lines with a high degree of aneupoidy and CIN with respect to abnormal spindle formation and malsegregation of selected chromosomes $(7,8,10,12,17$, and 18).

In total, we found three different kinds of chromosomal malsegregation likely representing different mechanisms leading to mitotic instability.

First, in most glioblastoma cell lines we found a bipolar spindle apparatus, but not all chromosomes were separated correctly during mitosis. Chromosomes 7 and 8 showed asymmetrical distribution of chromatids in a high percentage of investigated cells ( $75 \%$ and $44 \%$ respectively) (Fig. 1a) and b). Also chromosomes 10, 12, 17, and 18 displayed asymmetrical distributions in 14-28\% of investigated mitoses. Regarding the fact of unexpectedly high but significantly different percentages of non-disjunction for the different chromosomes (Tables II and III) an unknown chromosomespecific mechanism appears to be involved in the numerical aberrations of the investigated chromosomes. Also chromosome-specific failures of spindle attachment to the kinetochore or disturbed arrangement of chromosomes in the equatorial plane could result in chromosomal malsegregation.

The different frequencies of aneuploidy for the investigated chromosomes, detected by FISH-analysis in interphase nuclei and first interpreted to be the consequence of biological selection of cells containing distinct aneuploidies, have to be seen in a new light regarding the unexpected high frequencies and quantitative differences of de novo chromosomal malsegregation in mitotic tumour cells. 
However, selection effects cannot completely be excluded. Malsegregation should result in equal numbers of daughter cells with gain and with loss of the chromosome in question. The FISH data indicate that this holds true for only some of the chromosomes (e.g. 12, 17, 18), whereas for chromosome 7 nearly no monosomic and for chromosome 10 nearly no polysomic cells can be detected (6).

Second, one of the glioma cell lines studied (T5913) represents an additional kind of chromosomal malsegregation, i.e. mutlipolar spindles in more than one-third of the analyzed mitoses (Fig. 1 e and f). These multipolar spindle apparatuses appear to be functional, because all spindle poles develop microtubules which pull chromosomes to the respective spindle poles. In this case mitotic instability is caused by the supernumerary spindles, which hamper correct chromosomal segregation during mitosis indicating a chromosome nonspecific mechanism leading to mitotic instability in T5913. Likewise, Saunders et al described multipolar spindles in oral squamous cell carcinomas. In contrast to our results in T5913, they found that the poles of the multipolar spindle apparatus demonstrated different levels of chromosomal capture and alignment, indicating functional differences between the poles (5).

Third, in the breast cancer cell line MCF7 all analyzed mitoses showed a bipolar spindle apparatus intact in immunohistochemical analysis, but all investigated chromosomes were afflicted by a very high rate of missegregation in 30-40\% of the analyzed mitoses, indicating a chromosome non-specific mechanism in this cell line. Centrosome amplifications resulting in multipolar spindles were not observed. Yoon et al analyzed a series of breast cancer cell lines, including MCF7, and described that cell lines with high levels of chromosomal instability, like MCF7, have defective mitotic spindle checkpoints (21). Hence, defects in the mitotic spindle checkpoints are likely to cause the missegregation of all analyzed chromosomes observed in the MCF7 cell line.

Altogether, there are two general mechanisms that can cause aneuploidy. On the one hand, aneuploidy can originate from a single chromosomal malsegregation that persists throughout the following mitoses leading to an aneuploid cell clone with an advantage in selection. On the other hand, systematic malsegregation may result in aneuploid cells after each cell division. Because of the observation made in this study, we suppose the second type of mechanisms to be present in the glioma cell lines and in the MCF7 cell line. We found malsegregation of different chromosomes in many mitotic cells of all investigated cell lines. This could explain the high degree of aneuploidy observed in these cell lines.

A very interesting observation was the high frequency of mitoses with non-disjunction of both homologous partners of chromosome pairs. This results in a genomic separation of the respective chromosome, comparable to the mechanism of a first meiotic division. Due to the symmetrical distribution the chromosome number remains correct. This mechanism of genomic separation of a chromosome pair is not detectable by conventional cytogenetic analysis or interphase-FISH.

Fig. 2 elucidates schematically the different kinds of mitotic chromosomal segregation observed in cells showing a bipolar spindle apparatus. Fig. 2a displays a correct separation of chromatids, resulting in a symmetrical distribution. Four chromatids each are pulled to the respective spindle pole. Fig. 2b shows an asymmetrical distribution of separated chromatids. Fig. 2c and d display non-disjunction of sister chromatids resulting in an apparently symmetrical (Fig. 2c) or in an asymmetrical distribution (Fig. 2d). The mechanism shown in Fig. 2c does not lead to a visible aneuploidy, but it results in severe genetic imbalances because of homozygosity of regulatory genes.

The presented defect of centromere separation represents a new until now rarely discussed kind of mitotic chromosomal instability in tumour cells. It results in two daughter cell clones with genomic separation for the genes of a complete chromosome. The consequence is homozygosity for all heterozygous genes on this chromosome pair. It leads to restoration or homozygous loss of tumour suppressor genes and gene dose variation for oncogenes.

\section{Acknowledgements}

The authors would like to thank U. Lindemann for her technical assistance with tumour cytogenetics. This work was supported by a grant of the DFG-Graduiertenkolleg 'Zelluläre Regulation und Wachstum' to Alexandra Klein.

\section{References}

1. Lengauer C, Kinzler KW and Vogelstein B: Genetic instabilities in human cancers. Nature 396: 643-648, 1998.

2. Jung V, Romeike BFM, Henn W, Feiden W, Moringlane JR, Zang KD and Urbschat S: Evidence of focal genetic microheterogeneity in glioblastomas by area specific CGH on microdissected tumor cells. J Neuropathol Exp Neurol 58: 993-999, 1999.

3. Teixeira MR, Pandis N and Hein S: Cytogenetic clues to breast carcinogenesis. Genes Chromosomes Cancer 33: 1-16, 2002.

4. Borel F, Loheu OD, Lacroix FB and Margolis RL: Multiple centrosomes arise from tetraploidy checkpoint failure and mitotic centrosome clusters in p53 and RB pocket protein-compromised cells. Proc Natl Acad Sci USA 99: 9819-9824, 2002

5. Saunders WS, Shuster M, Huang X, Gharaibeh B, Enyenihi AH, Peterson I and Gollin SM: Chromosomal instability and cytoskeletal defects in oral cancer cells. Proc Natl Acad Sci USA 97: 303-308, 2000.

6. Loeper S, Romeike BMF, Heckmann N, Jung V, Henn W, Feiden W, Zang KD and Urbschat S: Frequent mitotic errors in tumor cells of genetically micro-heterogeneous glioblastomas. Cytogenet Cell Genet 94: 1-8, 2001.

7. Limon J, Dal Cin P and Sandbreg AA: Application of long-term collagenase disaggregation for the cytogenetic analysis of human solid tumors. Cancer Genet Cytogenet 23: 305-313, 1986.

8. Boveri T: Zur Frage der Entstehung Maligner Tumoren. Jena: Gustav Fischer Verlag, 1914.

9. Cahill DP, Kinzler KW, Vogelstein B and Lengauer C: Genetic instability and darwinian selection in tumors. Trends Cell Biol 9: M57-M60, 1999.

10. Cahill DP, Lengauer C, Yu J, Riggins GJ, Willson JKV, Markowitz SD, Kinzler KW and Vogelstein B: Mutations of mitotic checkpoint genes in human cancers. Nature 392: 300-303, 1998.

11. Masuda A and Takahashi T: Chromosome instability in human lung cancers: possible underlying mechanisms and potential consequences in the pathogenesis. Oncogene 21: 6884-6897, 2002.

12. Lingle WL, Lutz WH, Ingle JN, Maihle NJ and Salisbury JL: Centrsome hypertrophy in human breast tumors: implications for genomic stability and cell polarity. Proc Natl Acad Sci USA 95: 2950-2955, 1998.

13. Pihan GA, Purohit A, Wallace J, Knecht H, Woda B, Quesenberry P and Doxsey SJ: Centrosome defects and genetic instability in malignant tumors. Cancer Res 58: 3974-3985, 1998. 
14. Lingle WL and Salisbury JL: Altered centrosome structure is associated with abnormal mitosis in human breast tumors. Am J Pathol 155: 1941-1951, 1999.

15. Duensing F and Muenger K: Centrosome abnormalities, genomic instability and carcinogenic progression. Biochim Biophys Acta 1471: M81-M88, 2001.

16. Lingle WL, Barrett SL, Negron VC, D'Assoro AB, Boeneman K, Liu W, Whitehead CM, Reynolds C and Salisbury JF: Centrosome amplification drives chromosomal instability in breast tumor development. Proc Natl Acad Sci USA 99: 1978-1983, 2002.

17. Ghadimi BM, Sackrett DL, Difilippantonio MJ, Schröck E, Neumann T, Jauho A, Auer G and Ried T: Centrosome amplification and instability occurs exclusively in aneuploid, but not in diploid colorectal cancer cell lines and correlates with numerical chromosomal aberrations. Genes Chromosomes Cancer 27: 183-190, 2000.

18. Shono M, Sato N, Mizumoto K, Maehara N, Nakamura M, Nagai E and Tanaka M: Stepwise progression of centrosome defects associated with local tumor growth and metastatic process of human pancreatic carcinoma cells transplanted orthotopically into nude mice. Lab Invest 81: 945-952, 2001.
19. Cimini D, Fioravanti D, Salmon ED and Degrassi F: Merotelic kinetochore orientation versus chromosome mono-orientation in the origin of lagging chromosomes in human primary cells. J Cell Sci 115: 507-515, 2001.

20. Cimini D, Howell B, Maddox P, Khodjakov A, Degrassi F and Salmon ED: Merotelic kinetochore orientation in a major mechanism of aneuploidy in mitotic mammalian tissue cells. J Cell Biol 153: 517-527, 2001.

21. Yoon D, Wersto R, Zhou W, Chrest FJ, Garrett ES, Kwon TK and Gabrielson E: Variable levels of chromosomal instability and mitotic spindle checkpoint defects in breast cancer. Am J Pathol 161: 391-397, 2002.

22. Seabright M: A rapid banding technique for human chromosomes. Lancet 2: 971-972, 1971.

23. Mitelman F (ed.): An International System for Human Cytogenetic Nomenclature (ISCN). Karger, Memphis, 1995. 